Articulo Original

\title{
Integración entre servicios de salud y comunidad. Evaluación cualitativa de servicios de primer nivel de atención de la ciudad de Corrientes.
}

\author{
Alberto C Palladino*, Silvia B D’Angelo
}

\section{RESUMEN}

La integración entre el equipo de salud y su comunidad de referencia es una de las condiciones operativas, y conceptuales, de la Atención Primaria de la Salud. Con el fin de evaluarla, se realizó una investigación en las Salas de Atención Primaria de la Salud de la Ciudad de Corrientes. Se utilizó un método exploratorio orientado desde una perspectiva interpretativa-cualitativa. 30 alumnos de la Carrera de Medicina de la Universidad Nacional del Nordeste fueron capacitados a tal efecto. Se realizaron observaciones in situ y entrevistas semi-estructuradas al personal de salud y a usuarios de las salas. Se realizó un análisis de contenido, esquematizado en un sistema de categorías preestablecidas y enriquecido con subcategorias inducidas a partir del análisis de la información. Como facilitadores para la integración, se encontró por parte del personal del equipo: conocimiento de los problemas de la zona, residencia en el barrio, buena accesibilidad a la atención y respuesta adecuada a la demanda; y por parte de la población: predisposición a participar. Como barreras para la integración: diferencias de conceptos respecto al rol del primer nivel de atención entre equipo de salud y comunidad y escasa integración de algunos profesionales. Se concluyó con que existen factores favorecedores importantes que habría que aprovechar y potenciar; en tanto, habría que trabajar sobre las barreras, comenzando por el rol fundamental que para esto tiene el equipo de salud. La mejor identificación de categorías que ha permitido este trabajo propicia la realización de estudios de mayor profundidad.

Palabras claves: atención primaria de la salud, primer nivel de atención, participación de la comunidad en salud, características del equipo de salud

\section{Summary}

The integration between the health team and its reference community is one of the operating and conceptual conditions, of the primary health care. In order to evaluate, an investigation was carried out in the services of primary health care of the city of Corrientes. An exploratory method oriented from an interpretive-qualitative approach was used. 30 students from the Medicine Career of the Northeast National University were trained for this purpose. In situ observations and semi-structured interviews were conducted with health personnel and services users. It was carried out an analysis of content, outlined in a system of preset categories and enriched with subcategories induced from the analysis of information took place. As facilitators for the integration, it was found by the staff of the team: knowledge of the problems in the area, residence in the neighborhood, accessibility to care and adequate response to the demand; and by the population: predisposition to participate. As a barrier to integration: differences in concepts regarding the role of the first level of care between health team and community and poor integration of some professionals. It was concluded that there are important predisposing factors that harness and promote; meanwhile, would have to work on barriers, beginning with the fundamental role that this has the health team. The best identification of categories that allowed this work promotes the realization of further studies.

Keywords: primary health care, primary care, community involvement in health, characteristics of the health team 


\section{Revista de la
Facultad de Medicina}

de la Universidad Nacional del Nordeste

Alberto C Palladino*, Silvia B D’Angelo Rev. Fac. Med. UNNE XXXV: 2, 24-32, 2015

\section{INTRODUCCION}

En las Asambleas Mundiales de la Salud de 1975 y 1976 se fijó la meta conocida como "Salud para todos en el año 2000". El enunciado (aparentemente utópico) hacía referencia a la posibilidad que debiera tener la población toda de acceder a la atención de su salud; la cual había sido ya declarada reiteradamente por la Organización Mundial de la Salud (OMS) como derecho insoslayable de todo individuo. La fijación de una fecha no era más que la expresión de que esa situación igualitaria debiera ocurrir en un futuro más o menos próximo. Sin embargo, la pregunta era ¿cómo lograrlo? Es así que surge la idea de la Atención Primaria de la Salud (APS), sobretodo impulsada por Halfdan Mahler (entonces director de la OMS). Sobre esa idea (compartida, inicialmente, por su par de UNICEF) se organizó y realizó una gran reunión mundial, que dió en llamarse: “Conferencia sobre Atención Primaria de la Salud y Participación Comunitaria" y que tuvo lugar en setiembre de 1978 en Alma Ata (Kasajastan, ex URSS). En reiteradas ocaciones, Mahler ha señalado que la APS, más que un conjunto de metas a corto plazo, "es una estrategia dentro del sistema de valores de Salud para Todos, que es la verdadera meta a alcanzar en el espíritu de justicia social"; y fue dado en el marco del reconocimiento de que un "nuevo orden económico mundial" más justo era imperativo (1). En el documento llamado Declaración de Alma Ata (2), se intenta transmitir los aspectos salientes de la idea. En el artículo 60 se ensaya una definición de APS que incluye características-valores de la misma cómo: "asistencia sanitaria esencial", "métodos y tecnologías prácticos, científicamente fundados y socialmente aceptables", "alcance universal", "plena participación", etc. Y en su artículo $4^{\circ}$ expresa: "El pueblo tiene el derecho y el deber de participar individual y colectivamente en la planificación y aplicación de su atención de salud". La participación como derecho supone la democratización del sistema de salud; en el que todo ciudadano (directa o indirectamente) tenga la posibilidad de expresar sus necesidades, sugerir sus preferencias de atención y monitorear y evaluar los resultados. Esto es un requisito indispensable en todo proceso de atención que adopte a la APS como estrategia principal. Lo es para todos los niveles de prevención y constituye un elemento clave en la promoción de la salud. Así fue definido en la 1a Conferencia Internacional sobre Promoción de la Salud, celebrada en Otawa en 1986 (3), al decir que "La promoción de la salud radica en la participación efectiva y concreta de la comunidad en la fijación de prioridades, la toma de decisiones y la elaboración y puesta en marcha de estrategias de planificación para alcanzar un mejor nivel de salud. La fuerza motriz de este proceso proviene del poder real de las comunidades, de la posesión y del control que tengan sobre sus propios empeños y destinos". David A. Tejada, quién jugara un rol trascendental en la concreción de la Conferencia de Alma Ata, expresó en un artículo escrito a propósito de los 25 años de aquella reunión: "Lo que sí fue muy claro, pero sigue siendo muy mal entendido, es la responsabilidad y el deber de todos - personas, grupos sociales y la ciudadanía en general- de cuidar de modo activo de su salud individual y colectiva. Lamentablemente, y en la práctica, las personas siguieron siendo consideradas como recipientes pasivos de actividades puntuales prestadas por sistemas de servicios institucionalizados de atención médica reparativa orientada a enfermedades específicas" (4). 
No hay dudas, -o al menos, no han sido argumentadas- de que la participación comunitaria es requisito sine qua non en un modelo de atención basado en la APS. Pero, para que la participación sea posible, debe existir una estrecha relación entre el equipo de salud y la comunidad. Y, aún antes que esto, debe ser posible el acceso a la atención. Una lamentable confusión conceptual entre APS y Primer Nivel de Atención (PNA) confina la primera a este último nivel; cuando la APS es una estrategia global de atención de la salud que atraviesa todo el sistema. Pero en verdad, la APS también tiene en al PNA, no solo su puerta de entrada, si no en el núcleo más importante del sistema; tal como lo expresa la Declaración de Alma Ata. Esta importancia está dada por el hecho de que esos atributos de accesibilidad universal a la atención, de participación de la comunidad y de integración equipo-comunidad son, funcionalmente, mejor y más plenamente realizables en el PNA. Es pertinente entonces, verificar la situación en que se encuentran la atención de la salud, desde la perspectiva de esas dimensiones si se desea verificar la vigencia de la APS como estrategia principal del sistema. La Ciudad de Corrientes, con una amplia red de PNA, fue el ámbito definido para esta investigación. La red de servicios de PNA está integrada por algunos de dependencia municipal (Salas de Atención Primaria de la Salud: SAPS) y otros de dependencia provincial (Centros de Atención Primaria de la Salud: CAPS). Desde hace muchos años los autores de esta investigación vienen trabajando en estos procesos de integración en diferentes proyectos con conclusiones que han sido presentadas en jornadas científicas de la región y el país (5-10). En estos trabajos se ha detectado, con preocupante insistencia, la percepción negativa y/o descalificadora de la población sobre el PNA de la Ciudad. En muchos casos, más preocupante aún, se expresa, directamente, por la ignorancia de su existencia o de sus posibilidades de atención. Un aspecto observado en el equipo de salud (que está sujeto a cambios y rotaciones frecuentes) es que no llega a consolidarse como tal y, menos aún, integrarse a la comunidad. En un trabajo que se asesoró recientemente (11), se indagó a pacientes de uno de los servicios de emergencia más importante de la ciudad sobre motivaciones para el uso de dichos servicios. Se observó que sólo un $6 \%$ de los pacientes concurría derivado desde un CAPS o SAPS, y el resto se había "autorreferido". El 18 \% dijo desconocer la existencia de esos servicios y un $33 \%$ manifestó concurrir directamente al hospital por considerar que allí se brindaba una atención de mejor calidad. En otro trabajo, realizado en el marco de una beca de investigación de pregrado (asesorada por los autores), se indagó sobre la satisfacción de usuarios de CAPS de Corrientes. Los resultados muestran que la casi totalidad de los encuestados en cuatro servicios de la Capital expresaron los inconvenientes en el acceso a la atención; sobre todo, por el sistema de turnos y los tiempos de espera (12).

La accesibilidad (acá, específicamente, al PNA) es la primera condición para que la atención se concrete. Y la participación de la comunidad es el atributo que permitiría la adecuación de esa atención a las necesidades reales y sentidas de la población. Para ambos es necesario que el equipo de salud y la comunidad de referencia estén suficientemente integrados. De esto se ocupó esta investigación; siendo el presente trabajo un informe parcial sobre las visitas a servicios de PNA, a Centros Integradores Comunitarios (CIC) y a miembros de la comunidad.

El objetivo del trabajo fue identificar algunas categorías de análisis que pudieran obrar como facilitadoras o como barreras de la accesibilidad y/o de la participación comunitaria y estas últimas, en consecuencia, como barreras para la integración ente el sector salud y la comunidad. 


\section{Material y métodos}

Siendo la integración un fenómeno complejo y no teniendo información previa, esta primera instancia de alcance exploratorio, se ha orientado desde una perspectiva interpretativacualitativa siguiendo el diseño de teoría fundamentada.

Se realizaron observaciones in situ particularmente de reuniones de las Mesas de Gestión de los CIC (MG), considerando que estas reuniones son un espacio propicio para la integración ya que en ella confluyen integrantes de la comunidad y representantes de las diferentes instituciones que forman parte del área de influencia del CIC para, en conjunto, priorizar las necesidades sentidas y definir soluciones. A partir de un muestreo intencional se realizaron entrevistas semi-estructuradas al personal de salud de las SAPS incorporadas al estudio y a los usuarios de dichas SAPS. Respecto a los usuarios, se realizaron entrevistas, en un primer momento a quienes se encontraban en las SAPS el día de la recolección de datos. En un segundo momento, la recolección de información se realizó mediante entrevista estructurada, casa por casa. Se buscó en esta etapa incluir la opinión de quienes no acuden a los servicio de salud del barrio o lo hacen con poca frecuencia.

Para la recolección de la información se capacitaron a 30 alumnos del segundo año de la carrera de Medicina de la Universidad Nacional del Nordeste (Argentina); quienes junto a docentes, realizaron visitas en el segundo semestre del año 2013 a las SAPS de los Barrios Anahí, Nuevo y Lomas y visitas a los espacios correspondientes a los CIC Anahí, Néstor Kichner y San Ignacio.

Posteriormente, se realizó un análisis en espiral de los datos recolectados, siguiendo el método de comparación constante; esquematizado en un sistema de categorias preestablecidas y enriquecido con un segundo nivel de subcategorias inducidas a partir del análisis de la información. El sistema categorial quedó configurado de acuerdo al esquema del Cuadro 1. La validación de los resultados se realizó mediante vigilancia epistemológica, triangulación de datos y saturación.

\section{Resultados}

En relación al personal de salud, se pudo observar un adecuado conocimiento del equipo de salud acerca de las características de la población usuaria de las tres SAPS observadas; lo cual es un facilitador para la integración. También, constituye un indicador positivo el hecho de que gran parte del personal residía en la zona; aunque, existía un grupo de profesionales nuevos (menos de un año desde su ingreso) que aún no conocía la población del área programática. Este personal correspondía a profesionales incorporados específicamente al área de los CIC en el marco del Programa de Médicos Médicos Comunitarios.

A fin de apreciar mejor la riqueza de las respuestas obtenidas de estos profesionales, así como de miembros de la comunidad, se transcriben textualmente algunas de ellas:

(Ec: Entrevista a personal de Programa Médicos Comunitarios; Eu: Entrevista a usuarios; Ep: Entrevista personal de salud)

Ec1: "...no conozco mucho porque hace poco que estoy acá y cuando vine no querían que salga de acá dentro, me decían que el barrio era peligroso..." "el relevamiento lo tiene la gente de la SAPS". 


\section{Revista de la
Facultad de Medicina}

de la Universidad Nacional del Nordeste

Alberto C Palladino", Silvia B D’Angelo Rev. Fac. Med. UNNE XXXV: 2, 24-32, 2015

Ec2: "...la información la tiene el odontólogo y no está ahora, él tiene el relevamiento que hizo...".

Como barreras psicosociales se observaron: diferencias conceptuales entre el personal de salud y la comunidad acerca de las funciones de los servicios; la participación entendida por una parte de los encuestados sólo desde su dimensión pasiva; diferencia en el concepto de integración entre el área de salud y la comunidad y de los roles de cada uno de los actores (servicios de salud, equipo de salud y comunidad). Algunas respuestas de miembros de la comunidad obtenidas:

Eu1: "... cuando estoy enfermo o no me siento bien, voy a la sala".

Eu2: "...en el CIC se hace prevención solamente, las enfermedades se tratan en la salita".

Y otras del equipo de salud:

Ep1: "Nosotros acá en la sala hacemos atención curativa y prevención. En lo que hace a actividades preventivas hacemos charlas sobre lactancia, prevención de caries, entre otras".

Ep2: "... no terminamos de entender que ya no somos una sala, somos un CIC pero no lo entendemos nosotros y tampoco ellos". (Hace referencia a la gente de la comunidad que trabaja en el $\mathrm{ClC}$ ).

Ep3: "La gente participa de nuestras charlas cuando las damos mientras esperan ser atendidos o a veces los invitamos a alguna actividad que organizamos y vienen".

Eu5: "Nosotros presentamos proyectos para prevención de problemas que son comunes en nuestro barrio pero la gente de la sala no quieren participar, nosotros los incluimos pero dicen que nosotros no podemos hacer eso".

Eu4: "La doctora no puede decir que no va a participar de los proyectos que nosotros hacemos,... ellos no pueden hacer reuniones aparte, pero así estamos, salud se reúne un día y nosotros otro día".

Ep1: "Ellos no pueden decirnos cómo manejar nuestra sala".

Sólo hubo coincidencia en un caso:

Ec2: "Nosotros acá (hace referencia al CIC) hacemos prevención, no atendemos, a los que necesitan atención los derivamos a la sala, nosotros no estamos equipados, no tenemos medicamentos"

Eu1: "Acá (se refiere al CIC) no se atienden enfermedades, se hace prevención, si alguien está enfermo tiene que ir a la salita o al CAPS ahí los doctores están para atender enfermedades"

En cuanto a características más asociadas a los servicios de salud un facilitador observado fue la accesibilidad geográfica, la cual no presenta mayores dificultades, excepto en los días de lluvias abundantes, cuando las calles se tornan un poco difíciles de transitar. Otro facilitador observado en los servicios de salud fue la atención a la demanda. Ambas situaciones fueron manifestadas tanto por el personal sanitario como por los usuarios. También hubo coincidencia entre el personal del equipo de salud entrevistado y los usuarios en algunas dificultades de tipo funcional, como el poco personal en relación a la demanda; lo que crea insatisfacción entre los usuarios y profesionales que se sienten sobrecargados.

Eu3: "A veces hay que esperar mucho porque hay pocos doctores o tenés que ir al CAP".

Ep3: "...y se hace cola porque viene mucha gente, nosotros somos pocos en la sala pero tratamos de cubrir todo". 
Otro factor que podría actuar como barrera funcional es la desvinculación que tienen los profesionales del Programa Médicos Comunitarios con el personal de las SAP, ya que no dependen de los directores de las SAPS y, en algunos casos, tiene poco contacto con ellos.

Ep1: "En el CIC hay asignados tres comunitarios; pero no dependen de nosotros, estamos en contacto pero nosotros no podemos decirles qué hacer, no sé cuál es su tarea; ellos dependen de una coordinadora, igual a veces hacemos trabajos en conjunto que nosotros organizamos" Ec1: "Nosotros no tenemos director, tenemos una coordinadora que está en la oficina de comunitarios y solo hacemos prevención"

Respecto a la dimensión de la participación comunitaria, se observaron dos espacios: las SAPS y las MG. Los datos recabados en las SAPS, nuevamente, marcan coincidencia entre las respuestas otorgadas por el personal entrevistado y los usuarios. Las actividades que se realizan desde la SAPS con la comunidad y en la comunidad son de tipo preventivas y organizadas, planificadas y llevadas a cabo por el equipo de salud; el que define, en consecuencia, el tema prioritario. La comunidad solo participa de manera pasiva.

Ep4: "Los temas para las charlas los elegimos nosotros de acuerdo a lo que vemos que más se necesita, según la estación, en invierno hablamos más sobre enfermedades respiratorias, y asi" Ec1: "algunas veces fuimos a las escuelas a dar charlas... Ios temas los propusimos nosotros"

En tanto las observaciones realizadas en las reuniones de la MG arrojan como dato positivo la voluntad de participación de la comunidad. Se observó también, desde la comunidad, la preocupación por mejorar los sistemas de comunicación, participación e integración con las instituciones de salud de sus respectivas zonas sanitarias y con los propios vecinos.

Se observó, así mismo, escasa participación del personal de salud de las SAPS; en particular en el CIC Néstro Kirchner, en donde ninguna de las reuniones observadas estuvieron presentes el personal de las SAPS como tampoco los Médicos Comunitarios asignados al CIC. En las entrevistas, el personal de salud alega escasez de tiempo para asistir a las reuniones, superposición de horarios entre las reuniones y las otras actividades que realizan. Sólo uno de ellos manifestó no sentirse cómodo en las reuniones por el clima en que se desarrollan.

Ep1: "Lo que pasa es que no coinciden los horarios de reuniones con los días que yo puedo estar pero igual va alguien de la sala"

Ec1: "De ser posible trato de no estar, no me gusta cuando discuten como ayer por ejemplo que me contaron que se armó lío"

Conclusiones

Del análisis de la información surge que existen factores facilitadores de la integración tanto en los servicios, en el personal de los equipos de salud y en la comunidad que hay que reforzar. El conocimiento socio-epidemiológico que tiene el equipo de salud sobre su población asignada favorece la detección de necesidades y orienta hacia las actividades preventivas y de promoción de la salud posibles. Este conocimiento de la situación socio-sanitaria de la población a cargo es facilitado por la atención de la demanda y la residencia de gran parte del personal en el área programática de la SAPS en la que trabaja. No obstante, es conveniente la realización periódica de relevamiento en terreno, debiendo quedar un registro en el servicio para poder ser consultado por cualquier personal que lo requiera. La voluntad de participación que maniestan los integrantes de la comunidad, así como la voluntad de integración manifestada por el personal de salud son facilitadores que deben ser potenciados generando estrategias que puedan unir esas voluntades. 
Asimismo, existen barreras constituidas por factores modificables a mediano y largo plazo, como son: las concepciones diferentes referentes al proceso de salud-enfermedad, la manera de trabajar en salud, la participación comunitaria, el rol de la comunidad en el sistema de salud y en particular en un sistema basado en la APS.

Estos factores, facilitadores y barreras, son esquematizados en la Figura № 1.

Este trabajo ha permitido difinir mejor ciertas categorías de análisis sobre las que se continuará indagando a fin de profundizar el conocimiento sobre las mismas y poder generar estrategias para actuar sobre aquellos factores que están obrando como barreras para la integración entre el sector salud y la comunidad.

CUARO No 1: SISTEMA DE CATEGORÍAS Y SUBCATEGORÍAS UTILIZADOS EN LA INVESTIGACIÓN UTILIZADOS EN LA INVESTIGACIÓN SOBRE INVESTIGACIÓN ENTRE SERVICIOS DE SALUD Y COMUNIDAD. CORRIENTES. AÑO 2003

\begin{tabular}{|c|c|c|c|}
\hline \multirow[b]{2}{*}{ CATEGORÍA } & \multirow[b]{2}{*}{$\underline{\text { SUB-CATEGORÍAS }}$} & \multicolumn{2}{|c|}{ SEGMENTOS TEXTUALES } \\
\hline & & $\begin{array}{l}\text { EQUIPO } \\
\text { DE SALUD }\end{array}$ & USUARIOS \\
\hline Personal & $\begin{array}{l}\text { Características del personal (SAPS o } \mathrm{CIC} \text { ): grupo } \\
\text { ocupacional, funciones, residencia. }\end{array}$ & & \\
\hline Centro de salud & $\begin{array}{l}\text { Características funcionales del centro de salud: } \\
\text { servicios que presta, horarios de atención }\end{array}$ & & \\
\hline Accesibilidad. & Facilitadores y barreras percibidas. & & \\
\hline Población & $\begin{array}{l}\text { Características socio- epidemioló-gicas de la } \\
\text { población asignada y usuarios efectivos }\end{array}$ & & \\
\hline Actividades & $\begin{array}{l}\text { Actividades que se realizan dentro y fuera del } \\
\text { servicio además de la atención curativa }\end{array}$ & & \\
\hline Relación centro/comunidad & $\begin{array}{l}\text { Opinión sobre la relación con la comunidad y motivos } \\
\text { de esa relación. }\end{array}$ & & \\
\hline Relaciones SAPS-CIC & Relación entre el personal de los SAPS y de los CIC & & \\
\hline Participación en gestión & Participación de la Mesa de Gestión del CIC & & \\
\hline $\begin{array}{l}\text { Valoración de las funciones } \\
\text { del servicio }\end{array}$ & $\begin{array}{l}\text { Opinión sobre las funciones que cumple y que debe } \\
\text { cumplir el centro de salud }\end{array}$ & & \\
\hline $\begin{array}{l}\text { Propuestas de mejoras para } \\
\text { el servicio. }\end{array}$ & $\begin{array}{l}\text { Propuesta de mejoras para el funcionamiento del } \\
\text { servicio }\end{array}$ & & \\
\hline Propuesta de actividades & $\begin{array}{l}\text { Propuesta de actividades que se podrían realizar en y } \\
\text { con la comunidad }\end{array}$ & & \\
\hline $\begin{array}{l}\text { Factores facilitadores y } \\
\text { limitadores observados por } \\
\text { el personal y por la } \\
\text { comunidad. }\end{array}$ & $\begin{array}{l}\text { Opinión sobre factores que puedan estar facilitando o } \\
\text { limitando la integración con la comunidad y } \\
\text { propuestas de mejoras }\end{array}$ & & \\
\hline
\end{tabular}


FIGURA 1: ESQUEMA DE FACILITADORES Y BARRERAS PARA LA INTEGRAGION ENTRE SAPS Y COMUNIDAD ENCONTRADOS A PARTIR DE OBSERVACIONES Y ENTREVISTAS AL PERSONAL DE LAS SERVICIOS DE ATENCIÓN PRIMARIA DE LA SALUD Y SUS USUARIOS. CORRIENTES, 2013.

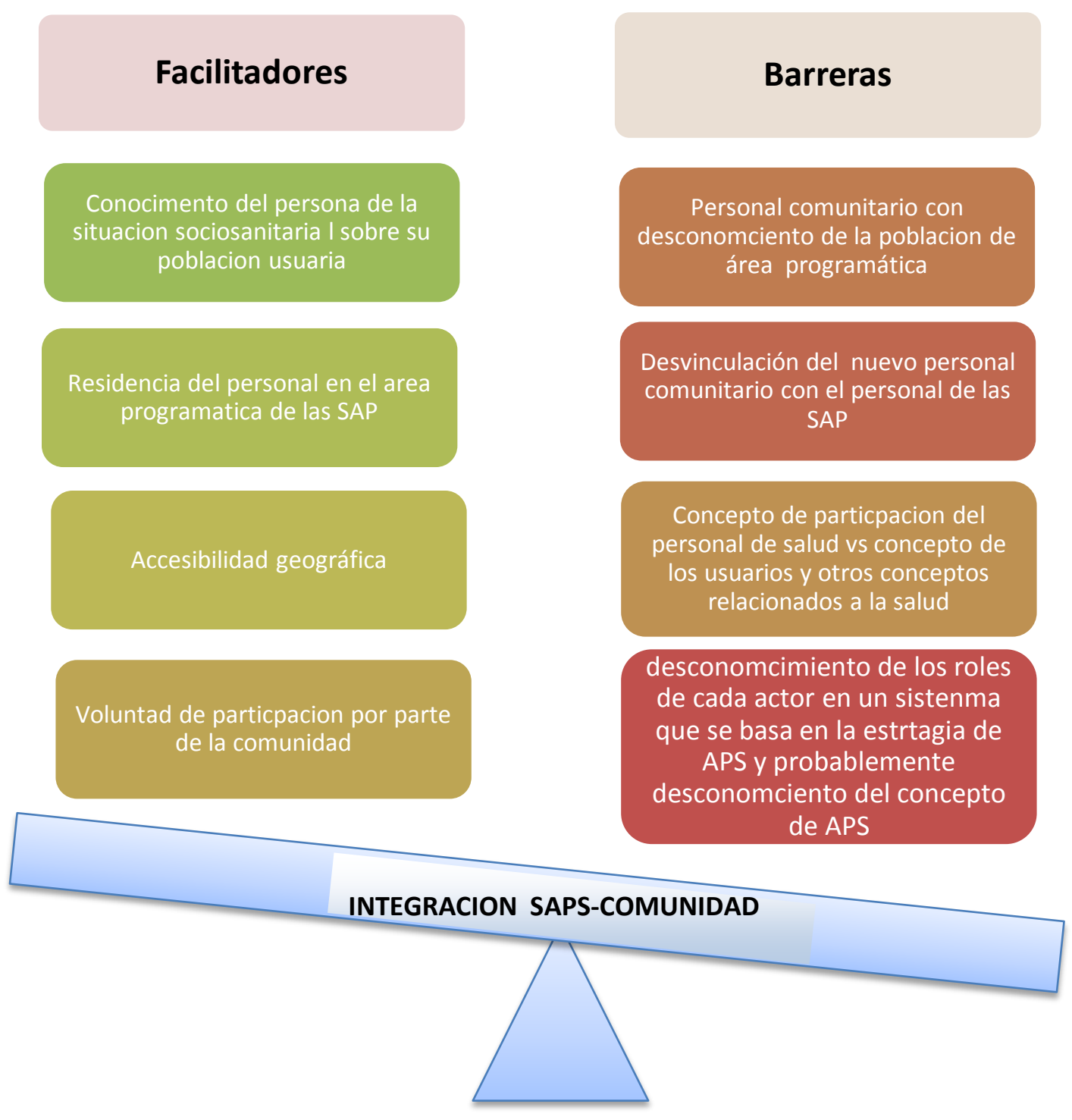




\section{0

\section{Bibliografía}

1. Mahler, H. Entrevista. Boletín de la OMS. Octubre 2008, 86 (10), páginas 745-746

2. Organización Mundial de la Salud. Atención Primaria de la Salud. Informe de la Conferencia sobre Atención Primaria de la Salud. Alma Ata, URSS, 6-12 de septiembre de 1978. Ginebra: OMS. Serie: Salud para todos, № $1 ; 1978$.

3. Carta de Otawa. Primera Conferencia Internacional sobre Promoción de la Salud. Canadá; 21 de noviembre de 1986.

4. Tejada, DA. “Alma-Ata: 25 años después”. Revista Perspectiva de Salud. Vol 8 (1). Washington: OPS; 20003.

5. Zurita, AG, D’Angelo, SB, Palladino, AC, Rey, AH. "Evolución de la municipalización de los servicios de salud en Corrientes, Argentina". Presentado en las “Jornadas de Comunicaciones Científicas y Tecnólogicas 2003" de la UNNE.

6. Zurita, AG, Palladino, AC, Rey, AH. "Evolución de la municipalización de los centros de salud en la Ciudad de Corrientes". Presentado en las “Jornadas de Comunicaciones Científicas y Tecnólogicas 2006" de la UNNE.

7. Zurita, AG, Palladino, AC. "Evolución de la atención médica en el primer nivel de atención. Provincia de Corrientes. 2002-2008. Presentado en las “Jornadas de Comunicaciones Científicas y Tecnólogicas 2009" de la UNNE.

8. Palladino AC, Barán MT, Estévez, JR. “Desarrollo de las competencias interfronterizas. Una propuesta de investigación-acción en vigilancia epidemiológica en el espacio común Encarnación (Paraguay) / Posadas (Argentina). Presentado en las “Jornadas de Comunicaciones Científicas y Tecnólogicas 2003" de la UNNE.

9. Zurita, AG, Palladino, AC, Rey, AH, D’Ángelo, SB. "Desarrollo del sistema de en el espacio interfronterizo argentino-paraguayo". Presentado en las "Jornadas de Comunicaciones Científicas y Tecnólogicas 2003" de la UNNE.

10. Palladino AC, Zurita, AG. “Autopercepción de profesionales del Programa De Médicos Comunitarios de Corrientes acerca de logros y obstáculos en su implementación". Presentado en las "Jornadas de Comunicaciones Científicas y Tecnólogicas 2008" de la UNNE.

11. Cáceres, CA, Quintana, MA, Rodríguez Gacio, LN, Zeniquel, MA. "Motivación en la demanda de atención de salud en el servicio de emergencias del Hospital J.R. Vidal. Corrientes 2013. Presentado en la VIIa Jornadas Científicas de la Facultad de Medicina. Corrientes, noviembre de 2013

12. Bossata, M, Lara, SL, Portillo Ruffa, R, Recalde, M. “Consulta médica óptima y dificultades para su aplicación en los Centros de Salud de la Ciudad de Corrientes". VIIa Jornadas Científicas de la Facultad de Medicina de 2013.

\section{Datos de autor}

Integración entre servicios de salud y comunidad. Evaluación cualitativa de servicios de primer nivel de atención de la ciudad de Corrientes.

Integration between health services and community. Qualitative evaluation of services of first level of attention of the city of Corrientes.

Alberto C Palladino*, Silvia B D’Angelo

Cátedra "Atención Primaria de la Salud, Epidemiología e Informática II". Facultad de Medicina. Universidad Nacional del Nordeste. Moreno 1240. 3400 CORRIENTES.

*Autor responsable: palladinoalberto12@gmail.com

Título abreviado: Integración entre servicios de salud y comunidad. Short title: Integration between health services and community. 